High Energy Phenomena in Relativistic Outflows (HEPRO IV)

International Journal of Modern Physics: Conference Series

Vol. 28 (2014) 1460199 (4 pages)

(C) The Authors

DOI: 10.1142/S2010194514601999

\title{
MOPRA AND NANTEN STUDIES OF HESS J1825-137 NORTHERN CLOUD*
}

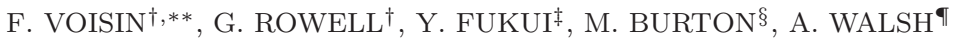 \\ and F. AHARONIAN $\|$ \\ †School of Chemistry and Physics, University of Adelaide, Adelaide, SA 5005, Australia \\ ${ }^{\ddagger}$ Department of Physics, University of Nagoya, Furo-cho, Chikusa-ku, Nagoya, 464-8601, Japan \\ $\S$ School of Physics, University of New South Wales, Sydney, NSW2052, Australia \\ IInternational Centre for Radio Astronomy Research, Curtin University, Perth WA, Australia \\ " Max-Planck-Institut für Kernphysik, Heidelberg, Germany \\ **fabien.voisin@student.adelaide.edu.au
}

Received 4 November 2013

Revised 25 November 2013

Published 21 March 2014

\begin{abstract}
The Pulsar Wind Nebula (PWN) HESS J1825-137 is one of the most extended TeV PWN and its morphology is influenced by the molecular gas located north of the TeV sources. In order to refine the composition and dynamics of the cloud, we have used the telescope Mopra in the 7 and $12 \mathrm{~mm}$ bands. Our results highlight dense regions and also perturbations in the southern part of the dense cloud.
\end{abstract}

Keywords: HESS J1825-137; PWN; PSR J1826-1334; ISM; diffusion.

PACS Numbers: 95.85.Bh, 98.58.Db, 98.70.Rz, 97.60.Gb, 98.70.Sa

\section{Introduction}

Located 3.9 kpc away from Earth, the 20 kyr old Pulsar Wind Nebula HESS J1825137 powered by the pulsar PSR J1826-1334 is one of the largest TeV source discovered by HESS. ${ }^{1}$ Its apparent assymetry is caused by a reverse shock, created by the interaction of a supernova shock with a dense molecular cloud, crushing the expanding PWN. ${ }^{2}$ The northern molecular cloud, located between $45-53 \mathrm{~km} / \mathrm{s}$ was revealed via the Dame CO survey by Lemière et al. ${ }^{3}$ Figure 1 displays the integrated intensity of the emission line $\mathrm{CO}(1-0)$ in this region between 41 and $55 \mathrm{~km} / \mathrm{s}$ as seen by the Nanten telescope, which surveyed the southern sky with a beam size

\footnotetext{
${ }^{*}$ This is an Open Access article published by World Scientific Publishing Company. It is distributed under the terms of the Creative Commons Attribution 3.0 (CC-BY) License. Further distribution of this work is permitted, provided the original work is properly cited.
} 


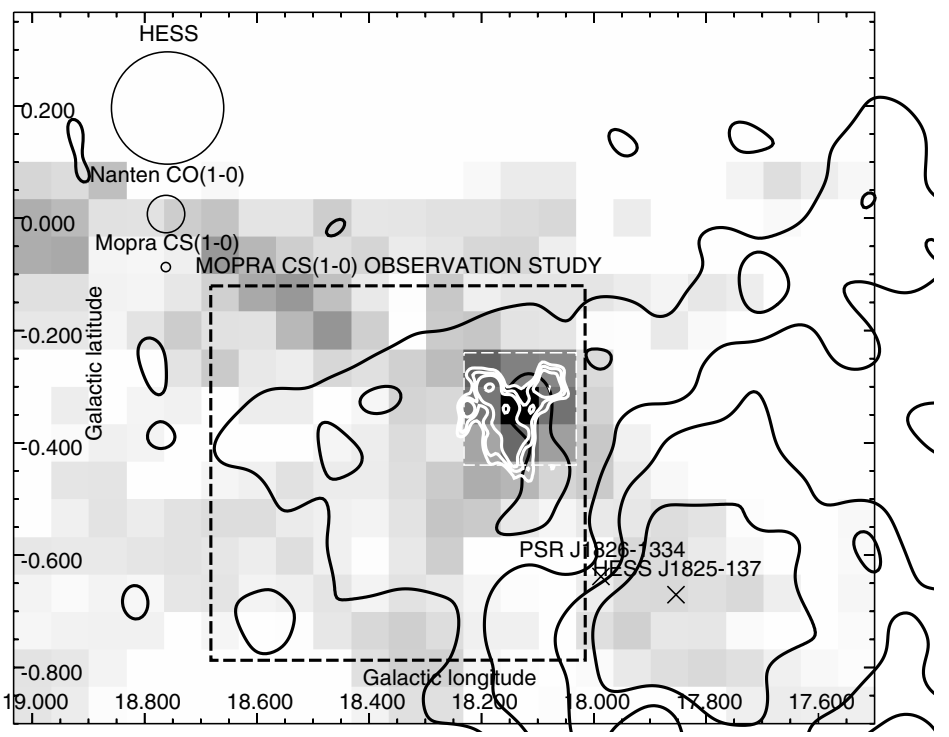

Fig. 1. Map of the region (in galactic coordinates) as seen by the Nanten $\mathrm{CO}(1-0)$. The black dashed box indicates the area observed by our Mopra CS(1-0) survey. The white contour displays the integrated intensity of CS(1-0) of the cloud of interest whereas the HESS TeV source is shown in black contours.

of 2.6'. Figure 1 shows the Nanten $\mathrm{CO}(1-0)$ image along with our Mopra observations of $\mathrm{CS}(1-0)$. Using the Nanten data and conversion factor ${ }^{4} \mathrm{X}=1.5 \times 10^{20}$ $\mathrm{cm}^{2}\left(\mathrm{~K} . \mathrm{km} . \mathrm{s}^{-1}\right)^{-1}$ between $\mathrm{CO}$ brightness temperature and the $\mathrm{H}_{2}$ column density, we derived the mass of the dense region encompassing the CS emission in white contours to be $1.1 \times 10^{5} \mathrm{M}_{\odot}$ (see white dashed box in Fig. 1.). Also, this region overlaps with the TeV emission presumably from HESS J1825-137. The PWN TeV emission is lepton dominated ${ }^{1}$ although the spatial overlap of the $\mathrm{TeV}$ emission and the ISM gas in the north may suggest a hadronic component. Such a hadronic component may come from the progenitor SNR and/or from the pulsar wind. ${ }^{5}$ Thus, we made use of the $\sim 1$ arc-min resolution 22 meter Mopra telescope and its ability to observe a wide range of spectral lines to refine the geometry, physical properties and dynamics of the molecular cloud located at the north of HESS J1825-137.

\section{Study of the Cloud with Mopra}

Mopra first observed the region in $12 \mathrm{~mm}$ as part of HOPS, ${ }^{6}$ and we continued observations in February 2012 and April 2013 in the 12 and $7 \mathrm{~mm}$ bands respectively. Then, several deep pointings in May 2013 allowed better quality spectra in key regions. Figure 2 displays the extended cloud ( 0.1 deg diameter) in integrated intensity as seen by the molecular lines $\mathrm{NH}_{3}(1,1), \mathrm{CS}(1-0)$, and $\mathrm{H} 62 \alpha$. The extended CS region has been observed encompassing the HII regions seen by Spitzer, also detected by the H62 $\alpha$ signal. We also observe that the CS(1-0) peaks do not appear 

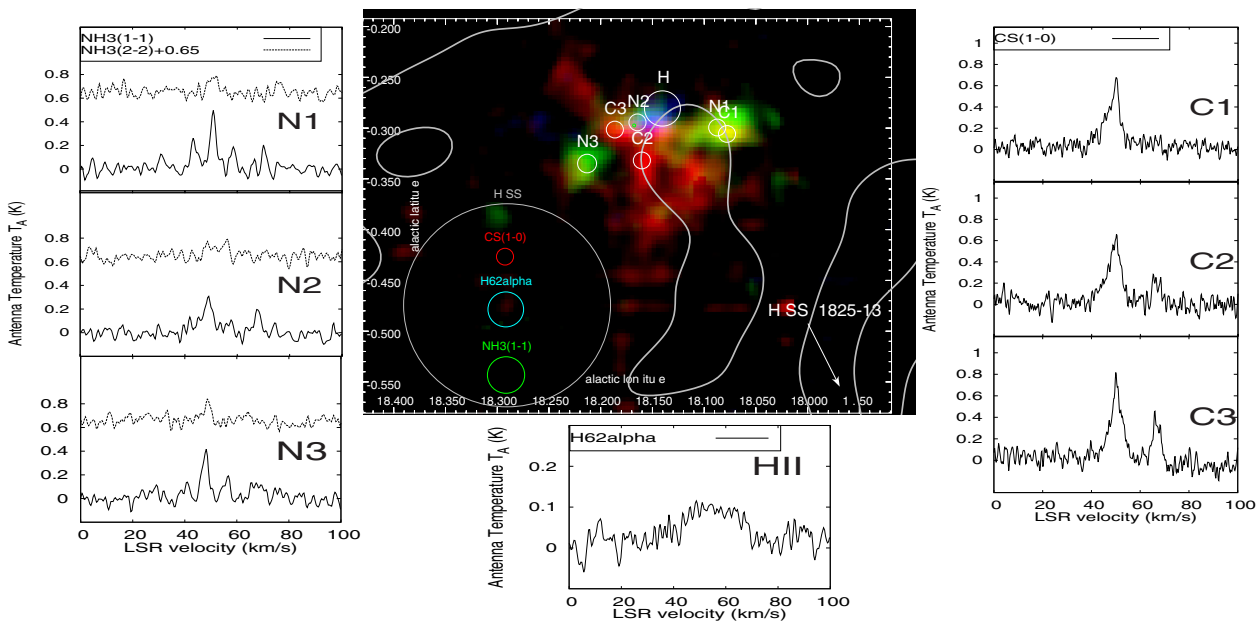

Fig. 2. Three colour map (in galactic coordinates) of the northern region of HESS J1825-137, showing $\mathrm{CS}(1-0)$ (red), $\mathrm{NH}_{3}(1,1)$ (green), and $\mathrm{H} 62 \alpha$ (blue) integrated intensity are displayed. The regions studied (where the signal was strongest) have been respectively named C[number], $\mathrm{N}$ [Number] for $\mathrm{CS}$ and $\mathrm{NH}_{3}$ regions. The HII region is detected by the recombination line $\mathrm{H} 62 \alpha$. The spectra of the respective regions are shown on the left for $\mathrm{NH}_{3}(1,1)$ and $\mathrm{NH}_{3}(2,2)$ (with an apparent offset value of $0.65 \mathrm{~K}$ ), on the bottom for $\mathrm{H} 62 \alpha$ and right for CS(1-0) (see online version for colours).

to spatially match with the $\mathrm{NH}_{3}(1,1)$ peaks. Assuming local thermal equilibrium, we estimated the $\mathrm{H}_{2}$ mass for the CS(1-0) emission to be $2 \times 10^{4} \mathrm{M}_{\odot}$. Figure 2 also displays the spectra of regions inside the dense complex having a centroid velocity of $49 \mathrm{~km} / \mathrm{s}$, with a kinematic distance of $3.9 \mathrm{kpc}$. Finally, we assume the gas detected by $\mathrm{CS}(1-0)$ to have a density close to the critical value of $3 \times 10^{4} \mathrm{~cm}^{-3}$. This density may enhance the $\gamma$-ray emission produced by any high-energy protons. More detailed studies of the cores will be described in a later publication. ${ }^{7}$

\section{Cloud Dynamics}

Figure 3 shows a complex structure in CS(1-0) where signals peak in three different velocity regions. The peaks located between 46 and $51 \mathrm{~km} / \mathrm{s}$ (green) are spatially separated from peaks located between $41-46 \mathrm{~km} / \mathrm{s}$ (red) and $51-56 \mathrm{~km} / \mathrm{s}$ (blue). From our deep pointing observations, Fig. 3 shows that the spectra closest to the pulsar (right) highlights two peaks with respective kinematic velocities of 43 and 53 $\mathrm{km} / \mathrm{s}$. However, the signal merges into a single wider peak at $49 \mathrm{~km} / \mathrm{s}$ (kinematic velocity of the pulsar) as we move further into the cloud. Also, the asymmetry of the spectra labeled as C1 and C2 (see Fig. 2) may indicate the directionality of a shock going through the cloud. Although this indicates turbulence inside the cloud, other shock indicators such as $\mathrm{SiO}(1-0)$ have not been detected. Further studies of the available mm-radio and IR data are planned to investigate the nature of perturbations and understand the relation between the $\mathrm{TeV}$ flux and the cloud. 


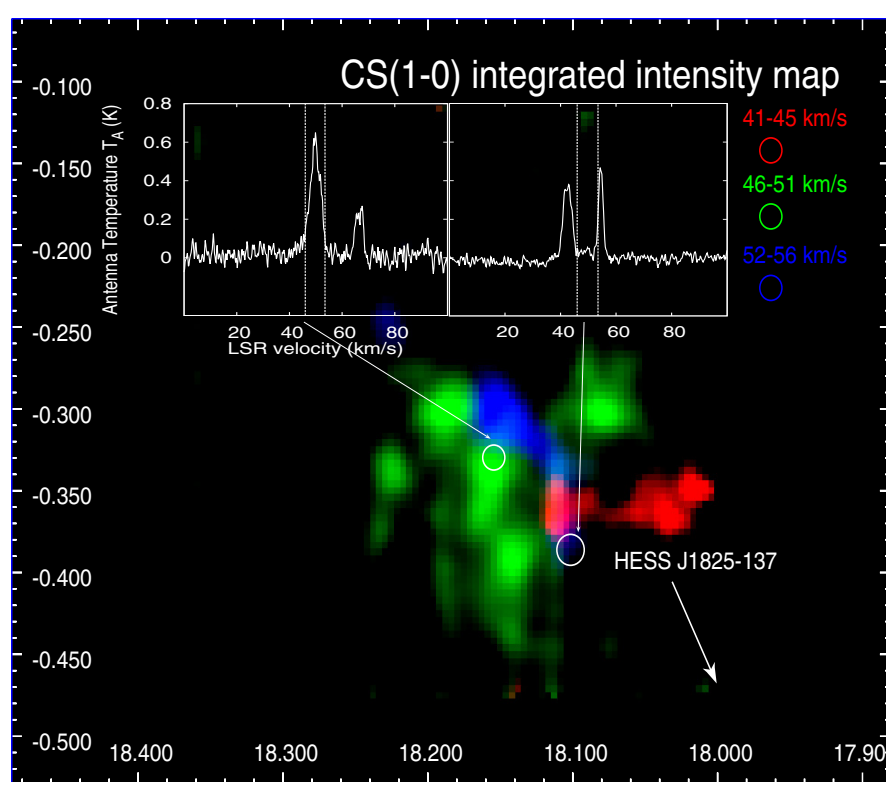

Fig. 3. Three colours map (in galactic coordinates) displaying the integrated intensity of CS(1-0) in $41-46 \mathrm{~km} / \mathrm{s}$ (red), $46-51 \mathrm{~km} / \mathrm{s}$ (green), $51-56 \mathrm{~km} / \mathrm{s}$ (blue) and the CS(1-0) spectra observed in 2 regions with Mopra. The white vertical lines represent the position and width of the signal in the left panel (see online version for colours).

\section{Summary and Conclusions}

We studied the molecular cloud north of the PWN HESS J1825-137 in the 7 and $12 \mathrm{~mm}$ bands with Mopra (and in $\mathrm{CO}(1-0)$ with Nanten) and detected extended CS emission surrounded by HII regions, and with broad and multi-peaked spectral lines indicating turbulence inside the dense cloud. These regions may provide suitable target sites for the enhanced hadronic $\mathrm{TeV} \gamma$-ray emissions and would be a good laboratory to study the diffusion of cosmic-rays inside the dense cloud. ${ }^{8}$

\section{Acknowledgments}

We acknowledge the support from Australia Research Council.

\section{References}

1. F.Aharonian et al. (H.E.S.S collab), A $6 A$ 460, 365A (2006).

2. J.M.Blondin et al., ApJ 563, 806 (2001).

3. A.Lemière et al., ICRC 4, 105L (2005).

4. A.W.Strong, I.A.W Moskalenko, O.Reimer et al., A $\& A$ 422, L47 (2004).

5. E.Amato et al., A\&SA 402,827(2003).

6. C.Purcell et al., MNRAS 426, 1972,(2012).

7. F.Voisin, G.Rowell et al. (2014), in prep.

8. S.Gabici et al., Astrophys. Space Sci. 309, 365 (2007). 